\title{
TTR
}

Traduction, terminologie, re?daction

\section{Langues sacrées et méthode de traduction}

\section{Jean-Claude Margot}

Volume 3, numéro 2, 2e semestre 1990

La traduction des textes sacrés : le domaine biblique

URI : https://id.erudit.org/iderudit/037066ar

DOI : https://doi.org/10.7202/037066ar

Aller au sommaire du numéro

Éditeur(s)

Association canadienne de traductologie

ISSN

0835-8443 (imprimé)

1708-2188 (numérique)

Découvrir la revue

Citer cet article

Margot, J.-C. (1990). Langues sacrées et méthode de traduction. TTR, 3(2), 15-31. https://doi.org/10.7202/037066ar d'utilisation que vous pouvez consulter en ligne.

https://apropos.erudit.org/fr/usagers/politique-dutilisation/ 


\section{Langues sacrées et méthode de traduction}

\section{Jean-Claude Margot}

\section{Introduction}

Aborder les problèmes soulevés par la traduction des textes sacrés (à savoir les textes bibliques en particulier), c'est être immanquablement amené à se poser la question préalable du caractère spécifique des langues originales dans lesquelles ces textes ont été rédigés (hébreu, araméen, grec): s'agit-il de langues sacrées à proprement parler, c'est-à-dire ayant une place tout à fait à part dans l'immense famille des langues de l'humanité? Autrement dit, l'expression «langues sacrées» est-elle vraiment pertinente? Une telle conception ne risque-t-elle pas, au contraire, d'engager le traducteur sur de fausses pistes, d'un point de vue aussi bien linguistique que méthodologique?

Sans prétendre apporter une réponse définitive à ces questions, je me propose cependant de formuler un certain nombre de remarques propres à mieux situer les enjeux du problème. Je le ferai en envisageant la notion de «langues sacrées» sous deux angles différents: 1) en considérant tout d'abord la structure même des langues bibliques; 2) en me référant ensuite à la terminologie traditionnelle adoptée au cours des siècles dans la plupart des versions bibliques, en Occident ou ailleurs. 


\section{Le caractère sacré des langues bibliques}

\section{a) Une conviction solidement ancrée}

Considérer que les langues bibliques sont sacrées est une conviction solidement ancrée dans la tradition des diverses confessions chrétiennes, et cela depuis longtemps (même si, comme nous le verrons plus loin, il est possible de trouver des avis divergents à cet égard). Il serait facile de démontrer cette constante à l'aide de multiples témoignages, tous fondés sur le raisonnement suivant: étant donné que les Saintes Écritures sont inspirées par l'Esprit de Dieu, on doit en déduire que le langage qui y est utilisé est un langage divin et, par conséquent, un langage qu'on ne saurait traiter comme les langues ordinaires des peuples de la terre. On en vient alors à parler d'un «langage du Saint-Esprit» ou même d'une «syntaxe du Saint-Esprit» ${ }^{1}$. Contentons-nous de quelques citations suggestives à ce propos.

Dans un dictionnaire technique de la foi chrétienne, paru en anglais en 1612 et dû à un certain Thomas Wilson, l'auteur de la préface, Roger Fenton (l'un des traducteurs de la Version autorisée, Authorized Version ou King James Version), s'exprime ainsi: «Bien que ce dictionnaire présente la définition du sens de mots et d'expressions, il s'agit pourtant d'un dictionnaire divin, enseignant en notre propre langue la langue du Saint-Esprit...» ${ }^{2}$. De son côté, un bibliste allemand du $19^{\circ}$ siècle, $\mathrm{H}$. Cremer, auteur d'un dictionnaire théologique du Nouveau Testament, emploie des expressions similaires dans la préface de son ouvrage: il a pour objectif, dit-il en substance, de montrer comment, dans la terminologie spirituelle du Nouveau Testament, la langue grecque subit en quelque sorte un bouleversement la rendant apte à devenir «l'organe de l'Esprit du

1. «Parfois, les caractéristiques (de la langue du Nouveau Testament) ont été considérées comme découlant directement de l'inspiration sacrée, de sorte qu'on attribua à cette langue dans son ensemble la qualification de langue du Saint-Esprit.» Carlo Buzzetti, La Bibbia e le sue trasformazioni (Brescia, Queriniana, 1984), p. 18.

2. Cité par J.-P. Dufour, Tradition et innovation, Recherches sur la traduction de la Bible, Version autorisée de 1611 (Université de Saint-Étienne, 1983), p. 187 
Christ» et de se muer ainsi en «langue du Saint-Esprit» ${ }^{3}$. Voici un autre exemple, attestant qu'une telle conception existe dans le monde catholique au même degré que dans le milieu anglican ou luthérien. Il s'agit d'un passage de la préface (préconciliaire, 1960) du Lectionnaire latin-français: "[on a voulu] autant que possible garder jusqu'à l'ordre des mots et la construction des phrases, puisque dans la Bible ce ne sont pas seulement des idées qui sont sacrées et inspirées, mais les mots eux-mêmes»".

Cette dernière citation est particulièrement suggestive pour notre propos, puisqu'elle montre expressément le lien direct qu'on établit entre la foi en l'inspiration des Écritures et la nécessité, pour le traducteur, de s'en tenir au calque en ce qui concerne aussi bien le vocabulaire que la syntaxe du texte original (avec la réserve, il est vrai, exprimée par les mots «autant que possible»). Il y a là, on le constate aisément, la justification, que l'on veut indiscutable, de la méthode de traduction dite «à équivalence formelle», largement enseignée, recommandée et appliquée dans le domaine de la traduction bibliques. Toutefois, dans la pratique, on est bien obligé de

3. D'après W.G. Kümmel, Das Neue Testament, Geschichte der Erforschung seiner Probleme (Freiburg/München, 1958), p. 243.

4. Cité par C. Orrieux, in V. Cosmao et al., Afrique et Parole, (Paris, Présence africaine, 1969), p. 32.

5. Selon Jean Delisle, «l'histoire de la traduction nous enseigne qu'un tel changement (à savoir une modification de la compréhension même de l'opération traduisante) s'est manifesté lorsque l'activité des traducteurs s'est déplacée de la traduction des textes religieux, qui avaient conditionné l'apparition de la «traduction mot-à-mot», à la traduction d'écrits profanes, qui ont donné le type de la «traduction de sens» au début de notre ère» (c'est moi qui souligne). Voir J. Delisle, l'Analyse du discours comme méthode de traduction (Presses de l'Université d'Ottawa, 1980), p. 237. De son côté, Umberto Eco, malgré son immense érudition, semble avoir ignoré ce phénomène lorsqu'il déclarait: «Quand Dante écrivait: Fede è sustanzia di cose sperate (la Foi est la substance des choses espérées), il utilisait les lois grammaticales et syntactiques du langage de son temps, pour communiquer un concept reçu par la théologie courante. Pour donner plus de prégnance à cette communication, il organisait les mots choisis avec soin, selon des lois imprévues et des rapports inédits. Il unissait de façon si étroite le contenu sémantique avec 
reconnaître que nul ne peut y recourir d'une manière tout à fait conséquente, car on est plus ou moins conscient de la nécessité d'éviter des barbarismes, des solécismes ou, tout simplement, des absurdités. C'est ce que laisse entendre, à côté de bien d'autres, l'un des auteurs de la Version dite de Lausanne, au siècle dernier, version qui se veut pourtant aussi littérale que possible: «Il est impossible, dit-il, de faire une traduction parfaite. Il faudrait, pour cela, avoir d'un côté la même grammaire, les mêmes formes, la même syntaxe, de l'autre, un vocabulaire exactement équivalent.» ${ }^{6}$ On sera assurément frappé par le caractère naïf de cette constatation, mais elle vaut la peine d'être mentionnée, car elle est typique de l'état d'esprit régnant chez maints traducteurs bibliques de tous les temps, y compris de l'époque contemporaine. Pour ma part, je me contente d'ajouter que le problème ainsi défini il y a plus d'un siècle saute aux yeux de manière encore plus flagrante dès qu'on aborde des familles de langues se caractérisant par des systèmes syntaxiques bien différents des nôtres, tout en ayant leur valeur propre.

Cela dit, il me semble opportun de présenter au moins deux illustrations des résultats auxquels peut conduire le «dogme» du calque, poussé presque à l'absurde ou, en tout cas, appliqué avec une ignorance complète des distorsions de sens qui en résultent dans la langue d'arrivée. Tout d'abord, en ce qui concerne le calque syntaxique, voici un exemple saisissant emprunté à un commentaire de l'épitre aux Éphésiens, paru en 1953. Suivant de très près la forme du texte grec, l'auteur donne la traduction (?) suivante d’Éphésiens 2.14-16:

les sons et avec le rythme général de la phrase, qu'il rendait celle-ci toute nouvelle, intraduisible...» Cette démonstration ne pourra convaincre que ceux qui ignorent que la phrase extraite de Dante est en réalité un calque du texte latin de Hébreux 11.1 (Est autem fides sperandorum substantia), lui-même calque du texte original grecl Voir U. Eco, l'Euvre ouverte (traduction française, Paris, Seuil, coll. «Points», 1979), p. 128.

6. L.-H. de Laharpe, cité par J.-C. Margot, Traduire sans trahir (Lausanne, l'Age d'homme, 1979; 1990, 2* éd.), p. 18. Bien entendu, ce que l'auteur de ce texte entend par «traduction parfaite» est une traduction qui serait un calque dans toutes ses parties. C'est ainsi que l'on confond bien souvent fidélité en matière de traduction et littéralisme. 
Car c'est lui qui est notre paix, lui qui des deux a fait un, et cela en abattant la séparation produite par la clôture, la haine, par sa chair. Il a abrogé la loi aux commandements s'exprimant en ordonnances, afin de créer en lui les deux en un seul homme nouveau, lui qui fait la paix, et de les réconcilier les deux en un seul Corps avec Dieu par la croix en tuant la haine en lui.

Dans une causerie présentée à un public international, il y a quelques années, j'étais en train de lire ce texte quand, soudain, l'interprète chargée de la traduction simultanée de français en anglais, a bondi hors de sa cabine pour me dire: «C'est incompréhensible! Comment voulez-vous que je traduise cela?» A quoi j'ai simplement répondu: «Voilà précisément ce que je désirais démontrer!» En effet, il est manifeste que si l'on veut donner quelque sens à une telle formulation, il convient de l'accompagner d'un commentaire détaillé. De plus, elle est la preuve évidente qu'un calque aussi étroit de la forme du texte original s'exerce au détriment du style en français, alors que la construction de la phrase grecque est naturelle, bien qu'un peu lourde.

Le second exemple se rapporte au calque du vocabulaire. Nombreux sont les traducteurs qui ont estimé, ou qui estiment encore, que, pour être fidèle au texte inspiré, il faudrait, dans la mesure du possible, rendre les mots du texte original toujours par les mêmes mots dans la langue d'arrivée (en quoi ils dénotent

7. Ch. Masson, l'Épître aux Éphésiens, Commentaire (Neuchâtel et Paris, Delachaux et Niestlé, 1953), p. 164. A titre de comparaison, voici ce que devient le même texte dans la version en français courant: «Car c'est le Christ lui-même qui nous a apporté la paix, en faisant des Juifs et des non-Juifs un seul peuple. En donnant son corps, il a abattu le mur qui les séparait et les rendait ennemis. Il a annulé la loi juive avec ses commandements et ses règlements, pour former de ces deux races un seul peuple nouveau, dans l'union avec lui; c'est ainsi qu'il a établi la paix. Par sa mort sur la croix, le Christ a détruit la haine; par la croix, il a réuni les deux races en un seul corps et les a réconciliées avec Dieu.» La Bible, traduite de l'hébreu et du grec en français courant (Paris, Alliance biblique universelle, 1982), p. $278 \mathrm{du}$ Nouveau Testament. 
assurément une ignorance totale de la polysémie des mots) ${ }^{8}$. Ainsi, dans la version Darby (du $19^{e}$ siècle, mais encore en usage dans certains milieux à l'heure actuelle), on a voulu rendre systématiquement le mot grec anthrôpos par «homme», alors qu'il peut signifier, selon les contextes, «homme», «être humain», «personne», «être intérieur». Il en est résulté que, dans une exhortation adressée aux femmes, en 1 Pierre 3.1ss., les auteurs de ladite version n'ont pas hésité à présenter ce texte surprenant: «...vous dont la parure ne doit pas être une parure extérieure qui consiste à avoir les cheveux tressés et à être paré d'or et habillé de beaux vêtements, mais l'homme caché du coeur...) (En grec, pour l'expression que je souligne, on a all' ho kruptos tês kardias anthrôpos.) Le lecteur non averti se demandera à juste titre quelle peut bien être l'identité de l'homme que la femme cache dans son cour (mari, amant...)! En fait, dans le texte grec il s'agit, sans hésitation possible, de l'opposition entre la recherche de l'apparence extérieure et celle des bonnes dispositions intérieures. Par conséquent, le sens à donner à anthrôpos, ici, est manifestement "être intérieur», "dispositions intérieures». Le cas particulier de ce terme grec est intéressant à un autre titre. En effet, bien loin de se rapporter exclusivement à l'homme en tant que mâle (que l'on désignerait plutôt par anêr, génitif andros, en grec), il désigne très souvent les êtres humains en général, sans distinction de sexe. C'est pourquoi, au lieu de le traduire par «hommes», comme on l'a fait la plupart du temps dans les versions bibliques usuelles, il vaudrait mieux employer «(êtres) humains», «gens», voire le féminin «(des) personnes» (et cela

8. Remarquons que cette tendance à traduire systématiquement certains mots du texte original par les mêmes mots dans la langue d'arrivée existe ailleurs que dans le domaine des textes sacrés, et cela pour diverses raisons. L'une d'entre elles est définie ainsi par Jean Delisle: «Deux vocables, l'un anglais, l'autre français, se ressemblant par la forme et le sens n'ont pas forcément la même fréquence dans leur système linguistique respectif. Par conséquent, l'emploi quasi systématique dans la version traduite d'équivalents sosies morphologiquement comparables (automatically étant traduit invariablement par "automatiquement», to affect, par "affecter», appropriate, par «approprié», problem, par «problème»...) a pour effet de garder dans l'ombre beaucoup de mots employés couramment et idiomatiquement par les usagers unilingues de la langue d'arrivée. C'est ce qu'on pourrait appeler le «réflexe morphologique».» C'est là un défaut parmi d'autres de ce que Delisle appelle «les traductions anémiques» (op. cit., p. 210). 
même si «homme» peut avoir en français le sens d'être humain en général: il s'agit d'éviter toute ambiguïté à cet égard). Telle est d'ailleurs la solution qui a été adoptée, là où elle s'imposait, dans la Version en français courant (à l'instar de ce qui s'est fait dans la Good News Bible, où un problème tout à fait semblable s'est posé à propos de l'emploi de man, men)' ${ }^{9}$. Comme cela m'a été dit, on évite ainsi (du moins en partie) de donner l'impression à certains et à certaines que nos traductions bibliques sont faites par des hommes pour des hommes!

En formulant quelques réserves à propos des exemples que j'ai cités, j'ai déjà laissé entendre que je trouve contestable l'application du concept de «langues sacrées» aux langues bibliques, avec les déductions qu'on en tire pour la méthode de traduction des Saintes Écritures. Mais il convient de motiver de façon plus précise la critique de cette conception, que nous avons simplement amorcée dans les lignes qui précèdent. Nous allons donc examiner maintenant sur quels arguments précis on peut fonder une telle critique.

\section{b) Langues sacrées ou langues courantes?}

Jusqu'à une époque récente, il était possible de considérer les langues bibliques comme des langues art, dans la mesure où l'on manquait de points de comparaison. Mais, depuis une centaine d'années, les nombreuses découvertes d'anciens documents ont permis de réviser sérieusement une telle vision des choses. En effet, les spécialistes ont pu se rendre compte que l'état des langues utilisées dans les textes sacrés (hébreu, araméen, grec) correspondait à celui du langage caractérisant le milieu dans lequel ces textes ont été rédigés. C'est ainsi que Moshe Greenberg, dans son introduction à l'hébreu biblique, déclare que la langue parlée par les Israélites au temps de la monarchie $\left(10^{\circ}\right.$ à $6^{\circ}$ siecle avant notre ère) était la «langue de Canaan» (voir Ésaie 19.18), et il ajoute:

La langue de la Bible était en fait un dialecte cananéen, ne différent que peu des langues parlées par les voisins d'Israël, les Phéniciens, les Moabites et les Édomites[...] La majeure partie de la littérature biblique est composée

9. Pour la version en français courant, voir la note 7 ci-dessus. Good News Bible with Deuterocanonicals/Apocrypha, The Bible in Today's English Version (New-York, American Bible Society, 1979). 
dans cette langue, et les quelques inscriptions extra-bibliques contemporaines découvertes par les archéologues en Palestine attestent que la langue de la Bible était en réalité d'un usage courant et non pas le produit d'une création littéraire artificielle. ${ }^{10}$ du ciel»!

On pourrait même dire «et non pas un langage descendu

Citons aussi le témoignage d'un éminent bibliste catholique, le Père $\mathrm{H}$. Cazelles, qui exprime un point de vue semblable:

De même que la découverte des papyrus grecs (à partir de la fin du $19^{\circ}$ siècle) a fait renoncer à l'idée qu'il y avait un «grec biblique» qui serait une langue sacrée originale, la koine, de même la découverte de la langue de Canaan utilisée par les lettres de Tell el Amarna, les tablettes d'Ugarit (14' siècle av. J.-C.) et même certaines inscriptions a fait s'évanouir l'idée que l'hébreu, son orthographe et son vocabulaire, pouvait constituer une langue particulière et sacrée... Les ressemblances ne se bornent pas au vocabulaire, mais concernent aussi le style, la prosodie et les modes du récit: dialogues ou répétitions... Les auteurs bibliques ont voulu avant tout être compris, et ils ont utilisé l'outil linguistique qui se présentait à eux.»"

Remarquons, à propos de cette dernière affirmation, qu'elle est dans la ligne de ce qu'ont écrit certains des anciens Pères de l'Église. Dans un ouvrage intitulé La Parole inspirée, le professeur Luis Alonso-Schoekel, lui-même traducteur de la Bible en espagnol, se réfère à plusieurs textes significatifs à cet égard. Relevons, par exemple, cette affirmation de saint Jean Chrysostome (fin du $4^{\circ}$ siècle de notre ère):

10. Moshe Greenberg, Introduction to Hebrew (Englewood Cliffs N.J., Prentice-Hall, 1965), p. 2s.

11. Cité par J.-C. Margot, op. cit., p. 37s. A propos de la découverte des papyrus grecs (principalement en Égypte) et de ses conséquences pour une meilleure évaluation du grec biblique, voir aussi C. Buzzetti, op. cit., p. 19s. 
Ne prenons pas à la légère ce qui est dit dans la Sainte Écriture, mais ne nous arrêtons pas non plus à la lettre; considérons plutôt qu'à cause de notre faiblesse, elle se sert de notre humble langage pour opérer notre salut d'une façon digne de Dieu... ${ }^{12}$

Quant à saint Thomas d'Aquin (13० siècle), il pose le principe suivant: «Dans l'Écriture les réalités divines sont traduites sous la forme que les hommes ont l'habitude d'utiliser. ${ }^{13}$ Autrement dit, pour s'adresser à ses créatures humaines, Dieu utilise les langues qu'elles parlent et qu'elles comprennent.

A l'évidence, de telles constatations sont appelées à jouer un rôle capital dans la mise au point d'une méthode de traduction biblique, qui soit justifiable aussi bien du point de vue linguistique que théologique. En effet, s'il est vrai que les langues bibliques sont celles que parlaient les humains à l'époque de la composition des textes sacrés, il en découle qu'elles sont susceptibles d'être traitées et analysées comme n'importe quelle autre langue humaine. Elles ne constituent pas un cas à part. C'est en se fondant en particulier sur ce fait que des théoriciens de la traduction biblique ont préconisé ce qu'ils appellent l'équivalence fonctionnelle (expression plus satisfaisante que celle d'équivalence dynamique employée précédemment) en opposition à l'équivalence formelle (ou calque des formes du texte original). ${ }^{14}$

Que faut-il entendre au juste par là? Sans vouloir entrer dans les détails, cherchons à y voir plus clair à l'aide de quelques données de base. Remarquons tout d'abord que l'opposition fonctionnelle/formelle pourrait induire en erreur, si elle laissait entendre

12. L. Alonso-Schoekel, la Parole inspirée (traduction française, Paris, Cerf, 1971), p. 36s.

13. Ibid.

14. Voir J. de Waard et E.-A. Nida, From One Language to Another, Functional Equivalence in Bible Translating (New-York, Thomas Nelson, 1986). 
au lecteur non averti que la fonction d'un énoncé $e^{15}$ serait entièrement distincte de sa forme. En réalité, il s'agit bien plutôt d'une révvaluation du juste rapport entre forme et fonction dans le processus de la traduction, en tenant compte du fait que ces deux notions sont également importantes. Pour établir ce rapport, nous bénéficions actuellement des enseignements de la linguistique générale ou descriptive. Cette science nous apprend à découvrir la façon dont chaque langue fonctionne, ou, autrement dit, à repérer les ressources dont elle dispose pour organiser un message quel qu'il soit. En effet, chaque langue a sa manière propre de le faire, chaque langue a ses structures syntaxiques ou sémantiques particulières. Par conséquent, la forme qui servira à exprimer un message dans une langue n'est pas la même que celle qui sera naturelle dans une quelconque autre langue, ou du moins pas forcément la même. Il peut arriver qu'il y ait coïncidence de forme et de fonction entre deux langues. Ainsi dans Genèse 39.7, si je traduis la proposition que la femme de Potiphar fait à Joseph par «Couche avec moil», bien que ce soit là une façon littérale de rendre l'hébreu, elle n'en est pas moins parfaitement significative en français dans une telle situation.) D'une manière générale, donc, l'essentiel pour le traducteur consiste à trouver dans la langue d'arrivée des formes ayant des fonctions équivalentes par rapport à celles de la langue de départ et non des formes identiques (contrairement à l'opinion de ceux qui s'imaginent qu'une traduction est d'autant plus «fidèle» qu'elle est plus littérale). Le simple calque des formes originales entraîne le plus souvent des distorsions de sens dans la langue d'arrivée, précisément parce que celle-ci ne fonctionne pas selon le même système que la langue de départ. Cela semble aller de soi pour le traducteur professionnel dans le domaine général de la traduction (littéraire, scientifique, etc.), mais c'est loin d'être évident pour beaucoup de traducteurs de la Bible.

De ce qui vient d'être dit, il serait erroné de déduire, comme on l'a fait parfois, que «peu importe la forme pourvu que le sens y soity! En réalité, il convient que le traducteur consciencieux se livre, dans le domaine biblique comme dans tout autre domaine, à une double analyse: a) d'une part, celle des formes du texte source, pour déterminer les fonctions précises auxquelles elles

15. Nous considérons ici le mot fonction dans un sens restreint, à savoir celle d'un énoncé ou d'une unité de sens, à distinguer de la fonction générale de la traduction biblique qui a pour objectif la communication fidèle d'un message considéré comme sacré. 
correspondent, du point de vue non seulement sémantique mais expressif (on se demandera, certes: «Que dit l'auteur?», mais en plus: «Comment le dit-il ? Pourquoi le dit-il ainsi et pas autrement? ${ }^{16}$; b) d'autre part, l'analyse des ressources de la langue d'arrivée devant permettre de découvrir quelles sont les formes propres à rendre les mêmes fonctions que celles du texte original. En résumé, on doit être constamment préoccupé de ne pas faire violence à la langue réceptrice en lui imposant des structures qui lui sont étrangères; mais, en même temps, on a le devoir de respecter le texte original en exprimant ses fonctions de manière aussi adéquate que possible, sans en négliger aucune.

Après ces quelques remarques critiques relatives à la notion de langues sacrées appliquée aux langues bibliques, il nous reste à examiner dans quelle mesure le langage traditionnel des versions bibliques mérite d'être considéré comme sacré, lui aussi, ainsi que le pensent beaucoup de fidèles.

\section{Le caractère sacré de la terminologie traditionnelle}

\section{a) Une longue tradition}

Dans les pays (occidentaux en particulier) où la traduction biblique a déjà une longue histoire derrière elle, on constate que les milieux intéressés sont en général très attachés à la terminologie traditionnelle, spécialement en ce qui concerne les mots clés. J'ai relevé ailleurs combien souvent ces mots clés ont leur origine dans le latin de la Vulgate, latin à proprement parler (expiation, rédemption, rémission, consommer au sens d'accomplir, etc.), ou grec latinisé

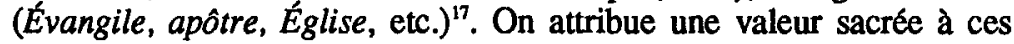
termes traditionnels; ils seraient intangibles: les remplacer par d'autres serait une vraie trahison, une hérésie (pour laquelle, autrefois, on aurait pu être condamné au bûcher...), et, de plus, une atteinte portée au caractère mystérieux du message des Saintes Écritures.

A la base de l'accusation de trahison ou d'hérésie, il y a, entre autres, la conviction que les concepts sont prisonniers d'un

16. Voir J.-C. Margot, op. cit., p. 336.

17. J.-C. Margot, «le Rôle des mots d'emprunts dans la traduction biblique», Meta, 35/1, mars 1990, pp. 188-194. 
certain vocabulaire: modifier le vocabulaire, ce serait perdre le contenu avec le contenant, ou, si l'on me permet l'expression, ce serait «vider l'enfant avec l'eau du bain», c'est-à-dire que, dans le cas particulier, on évacuerait des données fondamentales de la doctrine chrétienne. Or, une telle vision est infondée. En effet, dans n'importe quelle langue, il est toujours possible de recourir à des formes variées pour rendre une même idée (pensons au remplacement d'un terme par son synonyme dans un contexte donné, pour éviter une répétition; ou bien, songeons aux définitions du dictionnaire, qui expriment en d'autres mots les divers sens possibles des termes en cause). En outre, il y a plus grave: on ne tient pas compte du phénomène de l'évolution des langues, et très particulièrement de leur vocabulaire; on méconnaît le fait que ce qui pouvait être la manière précise de rendre le sens du texte original à telle ou telle époque, risque d'être fort mal compris, ou totalement incompris, par la suite, en raison d'un glissement de sens dû précisément à cette évolution.

On pourrait illustrer ce risque de malentendu à l'aide de multiples exemples. En voici quelques-uns: 1) Traduire le latin descendit ad infernos par «il (Jésus) est descendu aux enfers», comme dans l'ancien Credo, introduit en français toutes les connotations dont le mot «enfers» est chargé, au moins depuis le moyen âge (les damnés brûlant dans un feu activement entretenu par d'horribles démons...). Or, dans la perspective biblique, les «régions inférieures» du latin (car c'est cela qu'il signifie) désignent en réalité le séjour des morts, qui est totalement dépourvu de telles connotations. 2) La plupart de nos versions françaises, tributaires de la tradition, emploient le verbe «justifier» dans les épitres pauliniennes. Or, appliqué à l'œuvre de Dieu en faveur des humains, le verbe grec ainsi traduit signifie que Dieu les remet sur une juste voie, ou les rétablit dans une juste relation avec lui. En revanche, en français, «justifier» risque fort d'être compris au sens de «innocenter une personne, prouver que l'accusation portée contre elle est infondée», ce qui est évidemment contraire à l'enseignement de l'épître aux Romains sur la culpabilité, aux yeux de Dieu, de l'humanité dans son ensemble. Il y a donc là une source évidente de contresens, dont les spécialistes sont pour la plupart insuffisamment conscients. 3) L'expression «rendre grâces», typique d'un certain jargon ecclésiastique, est incomprise de beaucoup. Elle est en fait la traduction littérale du latin gratias agere (alicui), qu'il vaudrait mieux rendre aujourd'hui par «adresser des remerciements (à quelqu'un)» ou, tout simplement, par «remercier». 4) un cas amusant est relevé par Pierre-Jakez Hélias dans ses souvenirs d'enfance en 
Bretagne: à propos des leçons de catéchisme, il constate que le vocabulaire de la religion, en particulier le latin d'Église, est propice à certains malentendus. C'est ainsi, dit-il, que «nous (= les enfants) donnons notre assentiment total au Dies irae, dies illa ( Jour de colère que ce jour-là»). En breton, diêz veut dire difficile et nous sommes bien d'accord: tout cela n'est pas aisé, on a bien raison de répéter le mot.»! ${ }^{18}$

A la crainte de porter atteinte au sens si l'on modifie la terminologie traditionnelle s'ajoute celle de compromettre le caractère mystérieux du message des Saintes Écritures, ainsi que je l'ai déjà dit plus haut. A ce propos, on peut se borner à formuler une remarque: ce que d'aucuns appellent «mystère» est en réalité très souvent dû à une mauvaise traduction du texte source. En effet, là où le texte original comporte des tournures parfaitement naturelles en hébreu ou en grec, le calque qu'on en fait dans la langue d'arrivée a pour effet de présenter des tournures, insolites celles-là, difficilement compréhensibles pour le lecteur et par là même empreintes d'un soi-disant «mystère». Qu'on me comprenne bien: je ne prétends pas que le texte biblique soit dépourvu de tout mystère. Certains passages demeurent mystérieux à nos yeux en raison de la distance chronologique, linguistique ou culturelle qui nous sépare de leurs auteurs; dans d'autres cas, le "mystère» est manifestement voulu par l'auteur, il fait même partie du genre littéraire auquel il recourt (il suffit de penser au livre de l'Apocalypse, qui est en soi un exemple saisissant de ce fait). Toutefois, ce qu'il me parait essentiel de souligner pour mon propos, c'est une erreur que l'on commet trop souvent, à savoir celle qui consiste, en vertu de principes de traduction contestables, à introduire du «mystère» là où il n'a rien à faire.

Il est vrai que la volonté ou le goût de cultiver ce genre de «mystères» a existé à toutes les époques de la vie de l'Église. George Campbell, traducteur anglais d'une nouvelle version des Évangiles, à la fin du $18^{\circ}$ siècle, formulait une constatation significative à cet égard. Après avoir évoqué plusieurs dangers inhérents à une certaine conception conservatrice de la traduction, il ajoutait:

18. P.-J. Hélias, le Cheval d'orgueil (Paris, Plon, «Terre Humaine», 1975), p. 133. 
Un défaut supplémentaire de cette façon de traiter les Saintes Écritures réside dans le fait que les solécismes, barbarismes et expressions dénuées de sens qu'elle engendre, fournit abondance de matière pour le visionnaire, à partir de laquelle son imagination peut forger des milliers de mystères. ${ }^{19}$

Si l'on tient encore compte du fait que des exégètes amateurs ou professionnels aiment à détecter, par souci d'originalité, du mystère là où personne n'y aurait songé avant eux, on sera sans aucun doute convaincu que la nostalgie du mystère a un aspect psychologique indéniable. ${ }^{20}$

\section{b) Une tradition récente}

Jusqu'ici, j'ai évoqué la situation telle qu'elle se présente dans les pays où la traduction biblique a déjà une longue histoire. Mais, en ce qui concerne les problèmes posés par les mots clés, il faut également parler des régions où la Bible n'a été traduite que récemment, ou n'est que partiellement traduite. En effet, les difficultés dues à une terminologie considérée comme «sacrée» y sont tout aussi réelles, et voici pourquoi.

La plupart du temps, dans ces régions, on a commencé par traduire des textes liturgiques, que l'on utilise régulièrement lors

19. Cité par E.-A. Nida, Toward a Science of Translating (Leiden, Brill, 1964), p. 5.

20. L'anticléricalisme s'est d'ailleurs servi de l'argument selon lequel l'incompréhensibilité de certaines formules, donc leur «mystère», permettrait au clergé d'exploiter la crédulité du bon peuple des fidèles: «Le latin de la religion, un de ses palladiums, ressemble assez au Parapharagaramus des escamoteurs: ne pas comprendre est beaucoup pour le peuple... Le latin, c'est-à-dire l'inintelligible pour la masse, c'est l'arche sainte de la foi...» $E$. et $J$. de Goncourt, Journal (Paris, R. Laffont, «Bouquins», 1989) Tome I, pp. 187 (6 juillet 1856) et 236 (9 février 1857). De son côté, Julien Green (que l'on ne saurait traiter d'anticlérical!) note en date du 5 décembre 1984: «'C'est un mystère', me dit Parias. Le Moyen-Âge voyait toujours un mystère dans une obscurité. $\mathrm{Ce}$ serait, d'après lui, du rationalisme que de vouloir un éclaircissement.» J. Green, l'Expatrié, Journal 1984-1990 (Paris, Seuil, 1990), p. 49. 
des cultes, messes ou autres cérémonies religieuses. Or, lorsqu'on entreprend de traduire le Nouveau Testament dans son ensemble, puis toute la Bible, on répugne à recourir à d'autres termes clés que ceux déjà en usage. Évoquant le cas d'une traduction sud-africaine du début des années 1930, V.N. Webb constate que «la religion est pour beaucoup de gens essentiellement une expérience d'ordre émotionnel; il en résulte qu'ils sont tellement convaincus de la valeur religieuse de certaines formes qu'il leur est très difficile d'accepter qu'on les remplace par d'autres expressions ou d'autres termes dans une nouvelle traduction ${ }^{21}$. En ces quelques mots, Webb met le doigt exactement sur le nœud du problème.

Cependant, un tel «tabou» terminologique devient un obstacle presque insurmontable là où l'on forme des comités interconfessionnels, avec des traducteurs catholiques et protestants (de diverses dénominations). L'harmonisation de la terminologie technique de base, qui existe sous des formes très divergentes de part et d'autre, suscite des discussions longues et passionnées, parfois même dramatiques lorsque c'est la traduction dans son ensemble qui risque d'être mise en échec en cas de désaccord total sur un point particulier. Que faire, si chacun s'accroche farouchement à sa tradition liturgique, devenue «sacrée» à ses yeux? A la rigueur, on peut admettre que les jeunes générations seront plus ouvertes au changement; mais tel est difficilement le cas pour des gens plus avancés en âge, milieu auquel appartiennent en général les autorités ecclésiastiques prêtes à opposer leur veto à des décisions qui sortiraient des sentiers battus.

Ayant eu à affronter des situations de ce type dans des pays africains, voici la solution que j'ai préconisée en vue de sortir de l'impasse: je recommandais aux intéressés de faire une distinction entre la préparation de textes liturgiques ou catéchétiques, où le vocabulaire traditionnel a sa place, et celle de versions bibliques en langue courante, où le choix d'expressions plus simples devrait permettre d'apporter une lumière nouvelle sur des termes consacrés par l'usage ecclésiastique. En effet, il faut en être bien conscient, si ces termes ont une valeur émotionnelle indiscutable, il n'en est pas moins vrai qu'ils sont très souvent mal compris ou

21. V.-N. Weeb, «Some Aspects of the Sociolinguistics of Bible Translation and Exegesis, and of Religious Language», Sociolinguistics and Communication, ed. Johannes P. Louw (London, New York, Stuttgart, United Bible Societies, 1986), p. 77. 
carrément inadéquats par rapport aux usages actuels de la langue en cause $^{22}$. Par ailleurs, il est parfaitement admissible de disposer de traductions à divers niveaux, pour répondre aux besoins ou aux désirs du public cible (enfants, adultes habitués ou non au langage traditionnel...) ou à des circonstances particulières (liturgie officielle, lecture personnelle de la Bible.... ${ }^{23}$. S'en tenir à une position étroite et rigide dans ce domaine, c'est assurément tourner le dos à l'objectif visé, à savoir la communication efficace du message biblique.

\section{Conclusion}

Dans un article rédigé il y a une vingtaine d'années, Jacques Fédry, un jésuite français vivant au Tchad, écrivait ces lignes:

La Parole de Dieu, en s'incarnant dans une langue, va la travailler et probablement faire éclater les mots qui la diront. Sa radicale nouveauté s'exprimera non par l'introduction de vocables étrangers qui resteront extérieurs à l'expérience de l'auditeur, mais par la création d'énoncés inouïs, une réorganisation inédite des éléments de l'expérience, une «prédication» inusitée[...] $\mathrm{Du}$ coup, la nouveauté de ces énoncés, dans la mesure, bien sûr, où ils sont vérifiés par une expérience, va transformer les mots quotidiens employés jusqu'ici, leur «connotation» se chargeant d'un poids nouveau ${ }^{24}$.

A mon avis, il est quelque peu risqué de parler d'un «éclatement des mots», car c'est ouvrir la porte, qu'on le veuille ou non, à des expériences discutables, dans le sens de néologismes ou de la création d'un jargon, ce qui est d'ailleurs contredit par le reste du texte cité. En revanche, il est vrai que toute langue est assez souple

22. Voir J.-C. Margot, «la Bible qui divise, la Bible qui rapproche». Perspectives missionaires, 12/1986 (Bevaix, Suisse), p. 16s.

23. Voir J.-C. Margot, «Niveaux de langage», Traduire sans trahir, pp. 296-314.

24. J. Fédry, "Questions sur le problème théologique de la traduction de la Parole de Dieu», p. 17. (Cet article m'avait été remis par son auteur sous forme dactylographiée, mais je n'ai pas réussi à retrouver l'endroit où il a paru.) 
pour servir de support à des messages inédits ou «inouïs», ou pour s'adapter à des réalités nouvelles. Et ce qui est vrai d'une façon générale l'est aussi dans le domaine particulier de la traduction des textes sacrés, où, comme j'espère l'avoir montré, le traducteur n'est pas condamné à se laisser asservir par la notion de «langues sacrées». En bref, il est possible de communiquer dans une langue courante, naturelle, idiomatique, un message qui, lui, est sacré aux yeux de beaucoup, voire mystérieux dans certains de ses aspects. 